\title{
A New Regimen for Induction of Metabolic Syndrome and the Effect of Febuxostat in Ovariectomized Female Albino Rats
}

\author{
Rana Toghan ${ }^{\mathrm{a}}$, Rehab H. Abdel-Aziz ${ }^{\mathrm{a}^{*}}$, Tahany Abbas ${ }^{\mathrm{b}}$, Khaled A. Abdel-Sater \\ ${ }^{a}$ Department of Medical Physiology, Faculty of medicine, South Valley University, \\ Qena 83523, Egypt. \\ bepartment of Medical Histology, Faculty of medicine, South Valley University, \\ Qena 83523, Egypt. \\ ${ }^{c}$ Department of Medical Physiology, Faculty of Medicine, Al-Azhar University \\ (Assuit Branch), Assuit 71524, Egypt.
}

\section{Abstract}

Background: Metabolic syndrome (MetS) is increasing worldwide and correlates with serum uric acid and high fat combined with high fructose diet (HFFD). Fat raises triglycerides. Fructose increases uric acid. We hypothesized that HFFD in menopause may have a pathogenic role in MetS and using febuxostat may improve it by attenuating oxidative stress and vascular inflammation.

Objectives: This study aimed to assess effects of ovariectomy and HFFD diet on causing MetS for rats and effects of febuxostat on MetS of ovariectomized rats.

Materials and methods: The study was carried on 50 female white albino rats, aged 3 months and weighted 170 to $300 \mathrm{gm}$. 50 rats were divided into: sham control, ovariectomized control,HFFD non-ovariectomized, HFFD ovariectomized and febuxostat HFFD ovariectomized group. HFFD was composed of $45 \%$ fat and $30 \%$ fructose for 12 weeks. The latter group received febuxostat for 8 weeks.

Results: HFFD was associated with significant increased adiposity index (5.92 \pm 0.41$)$, serum triglycerides $(109.7 \pm 32.39 \mathrm{mg} / \mathrm{dl})$, HOMA-IR $(4.03 \pm 1.62)$ and serum uric acid $(5.99 \pm 0.528$ $\mathrm{mg} / \mathrm{dl}$ ). Ovariectomy has a synergistic effect with HFFD. HFFD with ovariectomy was also associated with significant increased adiposity index (11.49 \pm 1.5$)$,serum triglycerides $(223.2 \pm 67.6$ $\mathrm{mg} / \mathrm{dl})$, HOMA-IR $(11.48 \pm 2.01)$ and serum uric acid $(7.64 \pm 1.615 \mathrm{mg} / \mathrm{dl})$. Febuxostat was able to reverse these effects significantly in adiposity index (7.94 \pm 0.489$)$, serum triglycerides $(121.7 \pm 17.6$ $\mathrm{mg} / \mathrm{dl})$ and serum uric acid $(1.96 \pm 0.64 \mathrm{mg} / \mathrm{dl})$ but insignificant effect on HOMA-IR.

Conclusions: Fructose and fat in menopause may have a major role in incidence of MetS. Febuxostat prevent pathological condition of MetS by different mechanisms of action.

Keywords: metabolic syndrome, insulin resistance, hyperuricemia, fructose, febuxostat

\section{Introduction}

MetS is insulin resistance (IR) syndrome. It is defined as IR in inclusion to two of risk factors: obesity, hypertriglyceridemia, low high-density lipoprotein (HDL) cholesterol, hypertension, or microalbuminuria (Rochlani et al., 2017). It is about three times more common than diabetes; the global prevalence is around one quarter of the world population. Above a billion people were affected with MetS in 2018 in the world (Saklayen, 2018).
Patterns of food and diet changed during the last decades in both developing and industrialized countries together with sedentary lifestyle resulting in dramatic increases of obesity, MetS, non-alcoholic fatty liver disease and type 2 diabetes (Taskinen et al., 2019). MetS are becoming more common at a younger age due to increased consumption of sugar-rich and fatty-products. Fructose is used as a sweetening replaced of sucrose or glucose in the preparation of candy and carbonated beverages (Lozano et al., 2016). 
Menopause is the constant stopping of menses for 12 months. It is a normal physiological process that often occurs in women at an average age of 50 years. It is a risk factor for cardiometabolic diseases, MetS, type 2 diabetes and cardiovascular diseases. It is due to loss of the role of estrogens as protection and increased circulating androgens (Stefanska et al., 2015).

Uric acid is the final product of purine metabolism. Hyperuricemia isthe level of serum uric acid exceeds the normal range. Gout is induced by hyperuricemia. It also plays important role in MetS(Wang et al., 2018).

Non purine-like xanthine oxidase inhibitoris febuxostat used in the therapy of hyperuricemia.It leads to decreasing serum uric acid in humans not responding to allopurinol by decreasing xanthine oxidase $(\mathrm{XO})$. $\mathrm{XO}$ produce uric acid from hypoxanthine. Febuxostathas antioxidant effect and decreases reactive oxygen species (ROS) and oxidative stress, which is the main reason of inflammation of vessels in hyperlipidemia (Heikal et al., 2019).

In this study, we used febuxostat for treatment of albino rats model with postmenopausal MetS induced by ovariectomy and a high fat combined with high-fructose diet (HFFD). HFFD was used to induce MetS in rats, which closely mimics the natural pathogenic process of MS in human. There is a relationship between hyperuricemia induced by HFFD and MetS.

\section{Materials and methods}

\section{Chemicals and drugs}

Febuxostat was purchased from Hikma Pharma S.A.E., 6th of October City-Egypt. Fructose was purchased from Al-Gomhoria Pharmaceutical Co. (Cairo, Egypt).

\section{Animals}

This study is occurred in the animal house of Faculty of Medicine, Assiut University, Egypt. Fifty adult female white albino rats at first weighing from 170 to 300 grams and aged 3 months were gained from the animal house of Faculty of Medicine, Assiut University, Egypt. Rats were lived in clean stainless-steel cages $(42 \times 21 \times 20)$, natural light/dark cycle in a room infused with air and supplied with free entrance to food and water. All rats were adapted for one week before the experimental study was started. All procedures described in the present study were managed in correspondence with the Guide for the Care and Use of Laboratory Animals (Bayne, 1996) and were approved by the Ethical Committee for Scientific Research at the Faculty of Medicine, Assiut University, Assiut, Egypt.

\section{Experimental Design}

Rats were randomly divided into five groups (10 rats for each); group 1 (Sham group): adult rats subjected to laparotomy without removal of the ovaries, group 2 (control ovariectomized group): rats were anesthetized and bilaterally ovariectomized(small transverse skin incision of $2 \mathrm{~cm}$ wasmade with surgical scalpel blade on the middle part of the abdomen slightly towards right, just near to the second right nipple of the rat. The skin retracted to remove each ovary from the same skin incision. Then $2 \mathrm{~cm}$ peritoneal incision was made. After peritoneal cavity was accessed, the adipose tissue was pulled away until the right uterine tube and the ovary with its surrounding fat were identified. The ovary and associated fat were easily located and exteriorized by gentle retraction. The procedure was repeated for the left ovary through the same incision (Khajuriaet al., 2012)), group 3 HFFD nonovariectomized group), group 4 (HFFD ovariectomized group), group 5 (Febuxostat, HFFD - ovariectomized group). HFFD was composed of $45 \%$ fat (ram tail fat) and 30\% fructose for 12 weeks (Feillet-Coudray et al., 2019). Rats received Febuxostat $3 \mathrm{mg} / \mathrm{kg} /$ day orally via an orogastric tube for 8 weeks after taking HFFD for 12 weeks in group 5 (Omizo et al., 2020). The test drug febuxostat was dissolved in drinking water ad libitum at a concentration of $0.03 \mathrm{mg} / \mathrm{L}$ (Shirakura,2016).

Blood glucose determination and sample collection

At the termination of the study, rats were fasted overnight, and fasting blood glucose was determined with an automatic blood glucose meter (GlucoStar2 test strip, 
Japan) using blood samples from the tail vein. Rats were anesthetized with thiopental sodium (50 $\mathrm{mg} / \mathrm{kg}$ ) (Suckow et al., 2005) and sacrificed by decapitation at the end of the study. Samples of blood were collected by a cardiac puncture, centrifuged at $2000 \mathrm{rpm}$ for 15 min within $30 \mathrm{~min}$ of collection and stored at $-80^{\circ} \mathrm{C}$ until assayed.

\section{ELISA for insulin}

At the termination of the study, serum insulin was determined by using a rat insulin ultrasensitive an ELISA kit (Abnova, Jhongli, Taiwan) according to the manufacturer's instructions.

\section{Calculation of IR}

Homeostatic model assessment for IR (HOMA-IR) was determined IR by using this formula:

HOMA-IR index $=$ [fasting glucose $(\mathrm{mmol} / \mathrm{l}) \times$ fasting insulin $(\mu \mathrm{U} / \mathrm{ml})] / 22($ Er et al., 2016).

\section{Calculation of Adiposity index}

Body weight was measured with a top loader weighing balance (Model D0030, A \& D Company Limited, USA). The adipose tissue was dissected and then weighed. Total body fat was measured as sum of retroperitoneal fat + visceral fat+parametrial fat.

The adiposity index was calculated as (total body fat/final BW) $\times 100$. The adiposity index was used a tool for measure of obesity (Pimenta et al., 2019).

\section{Biochemical assays}

Serum uric acid and Serum triglycerides were determined at the end of the study, by Egyptian Company for Biotechnology (S.A.E) kits.

\section{Histopathological Examination}

The left lateral, the right medial, the caudate lobes of liver were used for histological examination. Samples were fixed in $10 \%$ Formalin, processed to paraffin, imbedded and cut in $5 \mu \mathrm{m}$ sections. Steatosis was evaluated in all slides with Mayer's Haematoxylin and Eosin (H\&E). Fibrosis and inflammation were evaluated based on the morphology on the H\&E stain. Examination was performed by using Olympus CX21 binuclar microscope.

\section{Statistical Analysis}

Statistical analysis was done suing the SPSS version 25 . Testing for normality of distribution of the data was done using Shaprio-Wilk the test. The data was illustrated in the form of mean SD and median with the 25 th and 75 th quartiles. To compare among all the 5 groups, Kruskal Wallis test was used and to compare between two groups, MannWhitney test was used. A P-value of less than 0.05 was considered significantly different.

\section{RESULTS \\ i.Effect of HFFD alone in induction of features of MetS}

Oral administration of HFFD for 12 weeks in HFFD non-ovariectomized group resulted in development of features of MetS manifested by significant increase $(p<0.05)$ in adiposity index, serum triglycerides, HOMAIR and serum uric acid with mean values of $5.92 \pm 0.41,109.7 \pm 32.39 \mathrm{mg} / \mathrm{dl}, 4.03 \pm 1.62$ and $5.99 \pm 0.528 \mathrm{mg} / \mathrm{dl}$ respectively as compared with sham group fed a control diet with mean values of $4.36 \pm 0.37,60 \pm 9.09$ $\mathrm{mg} / \mathrm{dl}, 0.49 \pm 0.303$ and $2.31 \pm 0.822 \mathrm{mg} / \mathrm{dl}$ respectively see figure $1,2,3$ and 4 .

ii.Effect of HFFD and ovariectomy in induction of features of MetS

Oral administration of HFFD for 12 weeks in HFFD ovariectomized group resulted in development of features of MetS manifested by significant increase $(\mathrm{p}<0.05)$ in adiposity index, serum triglycerides, HOMA-IR and serum uric acid with mean values of 11.49 $\pm 1.5,223.2 \pm 67.6 \mathrm{mg} / \mathrm{dl}, 11.48 \pm 2.01$ and 7.64 $\pm 1.615 \mathrm{mg} / \mathrm{dl}$ respectively as compared with control ovariectomized group fed a control diet with mean values of $4.29 \pm 0.414,61.8 \pm 8.76$ $\mathrm{mg} / \mathrm{dl}, \quad 0.51 \pm 0.277$ and $2.12 \pm 0.827 \mathrm{mg} / \mathrm{dl}$ respectively see figure $1,2,3$ and 4 .

\section{iii.Effect of febuxostat features of MetS}

Oral administration of febuxostat for 8 additional weeks in febuxostat HFFD ovariectomized group resulted in development of features of MetS manifested by significant decrease $(p<0.05)$ in adiposity index, serum uric acid and serum triglycerides with mean values $\quad$ of $7.94 \quad \pm 0.489,1.96 \pm 0.64 \mathrm{mg} / \mathrm{dl}$ and121.7 $\pm 17.6 \mathrm{mg} / \mathrm{dl}$ respectively as 
compared with HFFD non-ovariectomized group and HFFD ovariectomized group. On the other hand, HOMA-IR of febuxostat HFFD ovariectomized group did not differ significantly compared with HFFD ovariectomized group: $10.96 \pm 0.412$ vs. 11.48 \pm 2.01 respectively see figure $1,2,3$ and 4 .

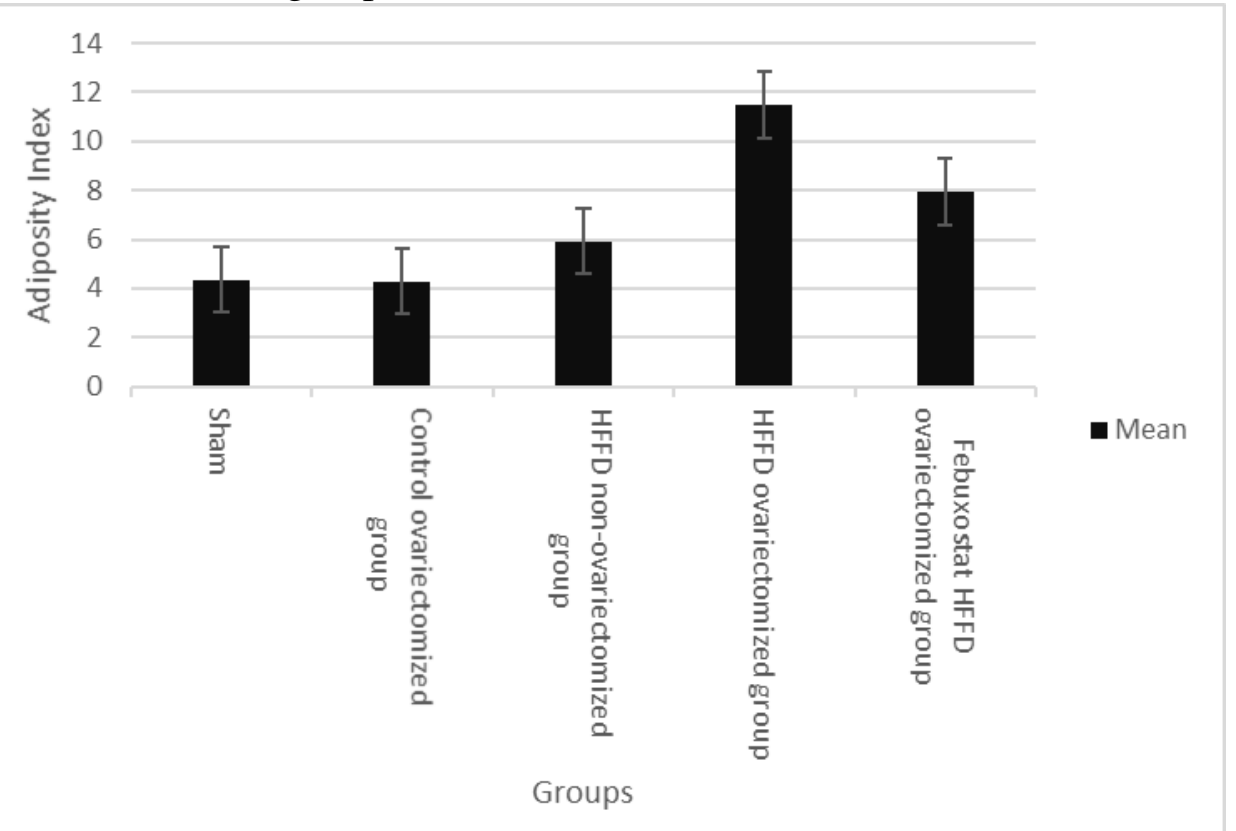

Figure 1. Mean adiposity index of experimental groups. Results are mean \pm SD

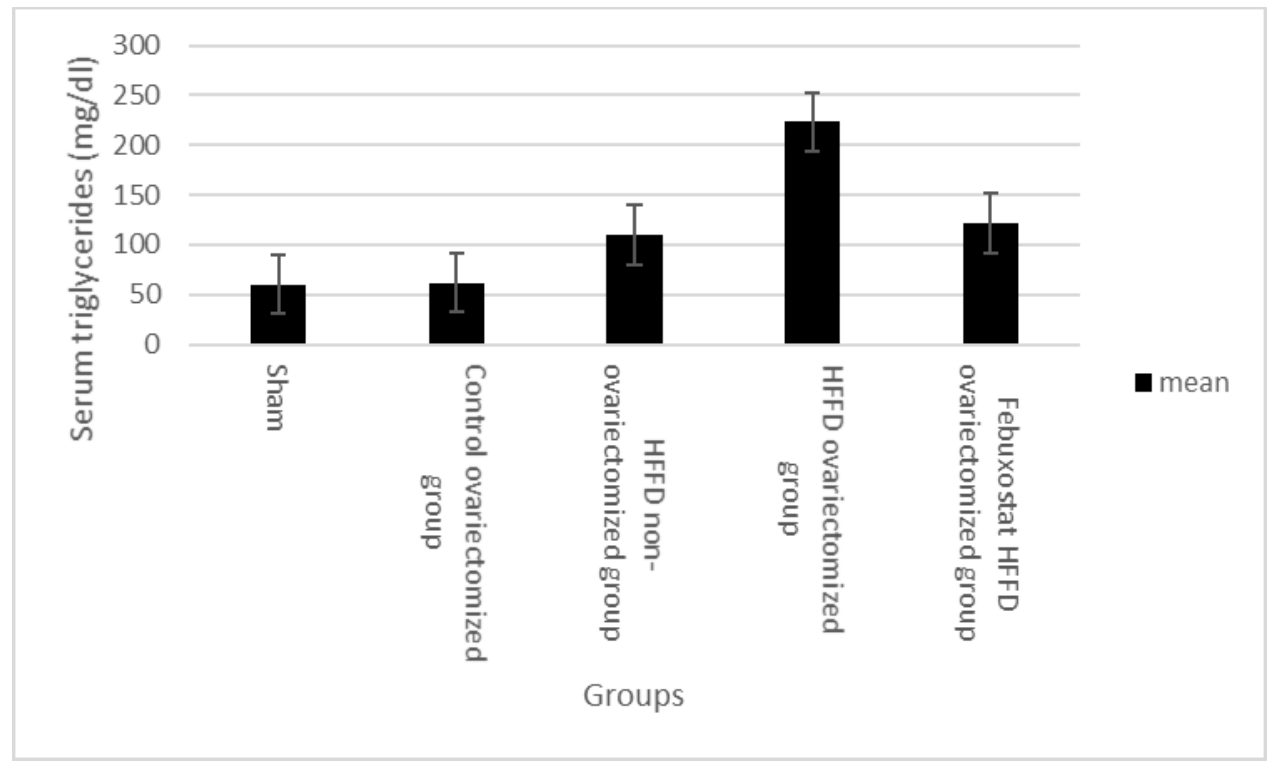

Figure 2. Mean serum triglycerides (mg/dl) of experimental groups. Results are mean \pm SD 


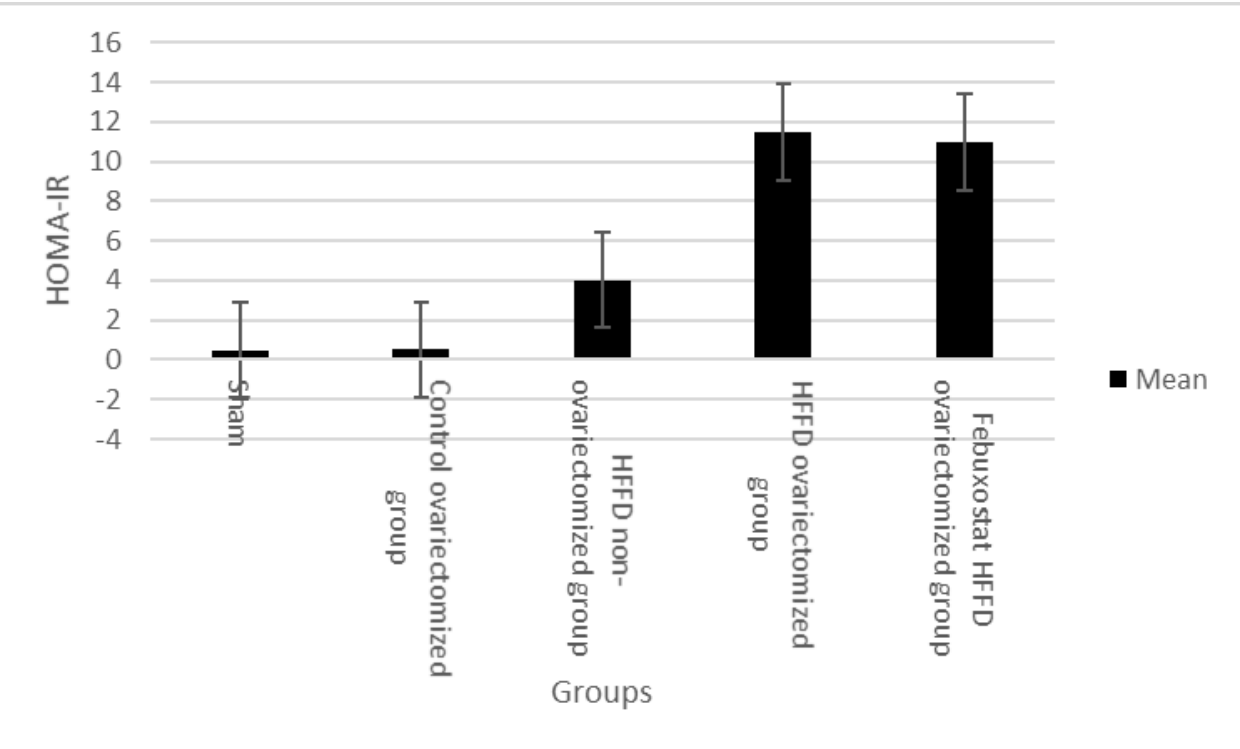

Figure 3. Mean HOMA-IR of experimental groups. Results are mean \pm SD

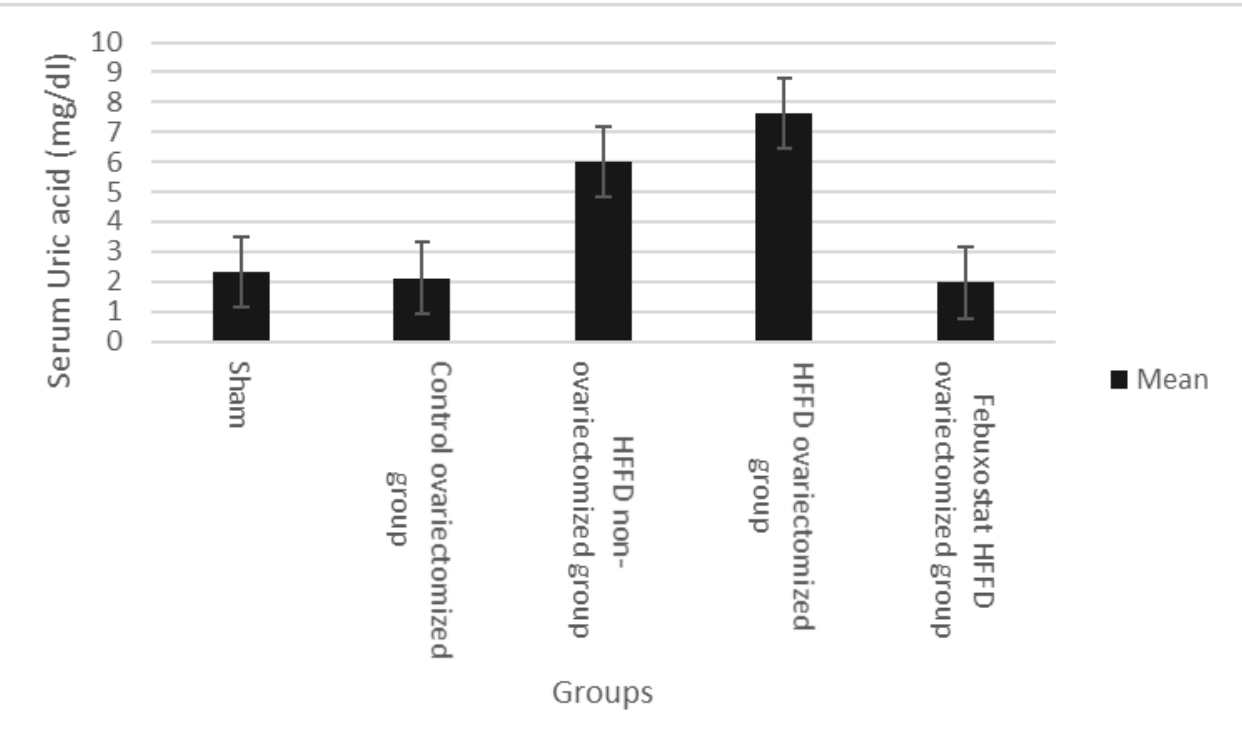

Figure 4. Mean serum uric acid (mg/dl) of experimental groups. Results are mean \pm SD 


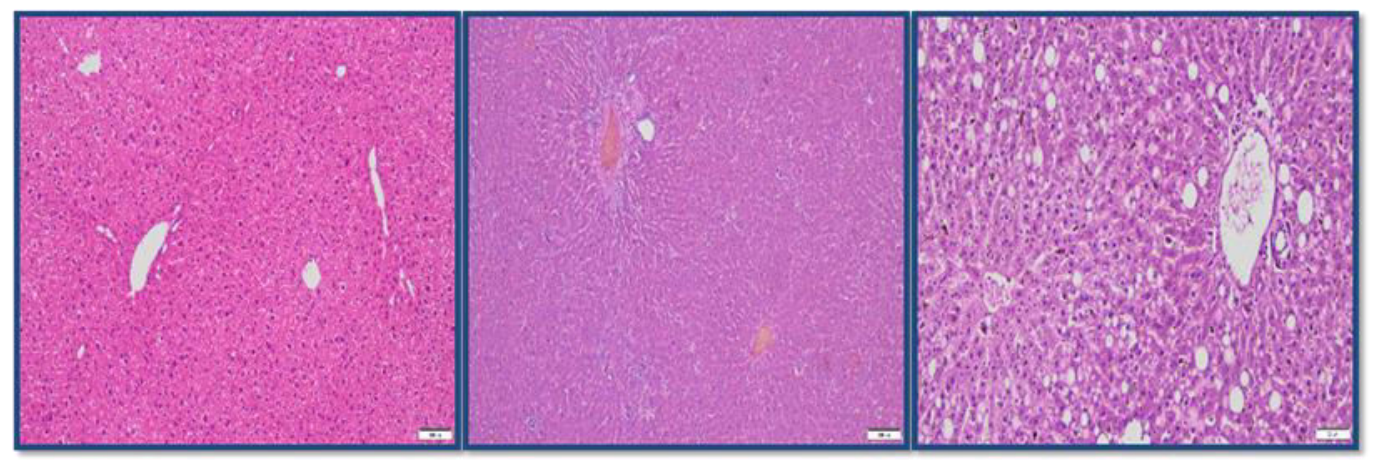

A) Control

B) Sham control

C)HFFD
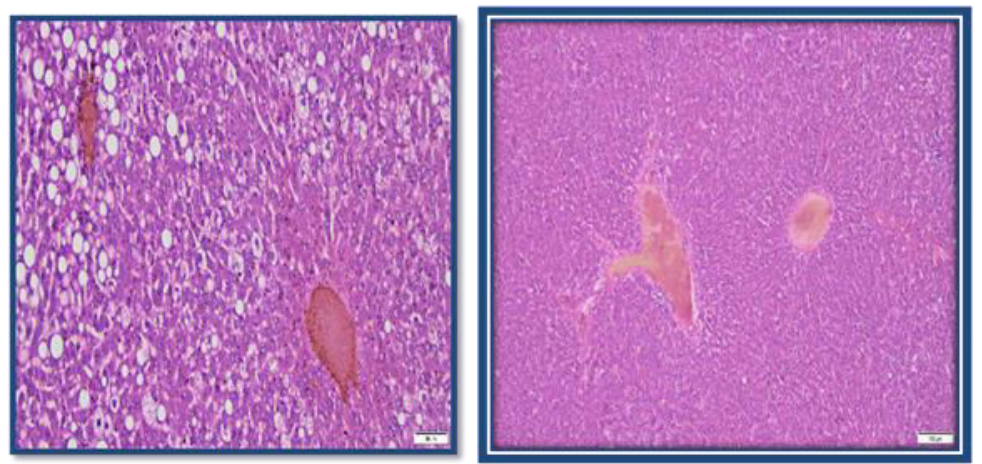

D)HFFD+Ovariectomy

E)Treated group

Figure 5: Hematoxylin and eosin staining of liver tissue sections of adult rats at different groups. The image (A) of a control group shows normal hepatic tissue. The image (B) of a sham control shows normal appearance of hepatic tissue. The image (C) of HFFD fed group shows mainly microvesicular steatosis and macrovesicular steatosis at the periportal area. The image (D) of HFFD ovariectomised group shows macrovesicular and microvesicular steatosis at the periportal area and zone2. The image (E) of treated group (received febuxostat for 8 week) show microvesicular steatosis in few hepatocytes at the periportal area.

\section{iv.Histopathological results}

Hematoxylin and eosin staining of liver tissue sections of adult rats at different groups. A control group shows normal hepatic tissue in figure 5A. A sham control shows normal appearance of hepatic tissue in figure $5 \mathrm{~B}$. HFFD non- ovariectomized group shows mainly microvesicular steatosis and macrovesicular steatosis at the periportal area in figure 5C. HFFD ovariectomized group shows macrovesicular and microvesicular steatosis at the periportal area and zone 2 in figure 5D. Feboxostat HFFD ovariectomized group for 12 weeks and then received febuxostat for 8 weeks) show microvesicular steatosis in few hepatocytes at the periportal area in figure $5 \mathrm{E}$.

\section{DISCUSSION}

We have succeeded in inducing MetS in experimental rats by administering $45 \%$ fat (ram tail fat) and 30\% fructose for 12 weeks in the diet. Features of the syndrome were in the form of obesity, hypertriglyceridemia, hyperinsulinemia and hyperuricemia. Also, liver steatosis or fatty liver is a hepatic manifestation of MetS (Cai et al., 2018). This method more closely imitates the pathogenesis happened in human MetS (Zhuhua et al., 2015).

In an attempt to know the effectiveness of febuxostat on attenuating features of MetS, febuxostat showed a significantly reducing obesity, hypertriglyceridemia and hyperuricemia and improving fatty liver only microvesicular steatosis in few hepatocytes at the periportal area in histological examination (the cytoplasm of hepatocytes is filled with 
tiny triglyceride droplets and nucleus is central in the cell). This could be attributed to the effect of febuxostat in attenuating oxidative stress and vascular inflammation that resulted from HFFD diet. Over-accumulation of lipids in hepatic cells causes oxidative stress. It increases expression of inducible nitric oxide synthase (iNOS) and elevation of NO production (Heikal et al., 2019). Also, Febuxostat had a significantly reducing hyperuricemia by inhibiting both the oxidized and reduced forms of XO. Febuxostat inhibits $\mathrm{XO}$ by binding to a long narrow channel that leads to the molybdenumpterin active site of the enzyme (Luna et al., 2019). On the other hand, and according to our study, it was found that febuxostat had insignificantly reducing hyperinsulinemia because high-sensitivity Creactive protein, tumor necrosis factor alpha or interleukin 6 is not decreased and then not effect on IR if duration of treatment of febuxostat less than 12 weeks (Drabsch et al., 2018). Febuxostat treatments for 12 and 24 weeks leads to a significant reduction in the levels of insulin and HOMA-IR by reduction of high-sensitivity $\mathrm{C}$-reactive protein because there is relation between inflammation and IR (Meng et al., 2017).

In our study, HFFD had a significantly increased obesity, hypertriglyceridemia and hyperuricemia and showing fatty liver as microvesicular steatosis and macrovesicular steatosis (cytoplasm of hepatocytes is filled with tiny triglyceride droplets or one large droplet and nucleus is peripheral in the cell) at the periportal area in histological examination. Fructose is phosphorylated into fructose 1phosphate by fructokinase in the liver during metabolism. This reaction leads to decreased levels of intracellular phosphate and ATP. After that, the enzyme fructose-1-p aldolase breaks fructose 1-phosphate into dihydroxyacetone phosphate and $\mathrm{d}$ glyceraldehyde. At a high intake of fructose, phosphorylation into fructose 1-phosphate is fast, but reaction with aldolase is slow so fructose 1-phosphate accumulates and intracellular phosphate decreases. This decrease stimulates AMP deaminase, which catalyzes the degradation of AMP to inosine monophosphate, increasing the rate of purine degradation. The purine degradation produces uric acid and mitochondrial oxidants. Mitochondrial oxidative stress inhibits aconitase in the Krebscycle, so it leads to accumulation of citrate and stimulation of ATP citrate lyase and fatty acid synthase leading to de novo lipogenesis (Caliceti et al., 2017).Also, we found that HFFD ovariectomized group had a significantly increasing obesity, hypertriglyceridemia and hyperuricemia and showing more fatty liver macrovesicular and microvesicular steatosis at the periportal area and zone 2 in histological examination when comparing with HFFD nonovariectomized group. In the postmenopausal status, female metabolism is disturbed leading to an increase in the propagation of hyperuricemia and MetS due to estrogen deficiency. Estrogen increases the renal excretion of urate (Techatraisak \& Kongkaew, 2017). Incidence of MeS is high in postmenopausal women. Estrogen prevents the conversion of angiotensin I to angiotensin II and decreases the sensitivity of angiotensin receptors. Renin activity, sympathetic activity and sensitivity to dietary sodium intake are increased in postmenopausal women. Estrogen acts on nitric oxide (NO) synthesis to promote vasodilatation by induction of endothelial nitric oxide synthase (eNOS) and stimulates calcium channel opening in smooth muscle cells (Das, 2016). The low sex hormone binding globulin levels is an important factor contributing to IR development in postmenopausal women. There are also low serum adiponectin levels causes IR. Adiponectin, an adipokine produced by fat, increases insulin sensitivity by promoting fat oxidation distally in liver and muscle (Kozakowski et al., 2017).

There is a relationship between uric acid and MetS. Uric acid is an antioxidant in low concentration, while in high conc it is a pro-oxidant. It induces mitochondrial translocation of the nicotinamide adenine dinucleotide phosphate oxidase 4 so leading to increased mitochondrial oxidative stress, mitochondrial dysfunction and citrate release to the cytosol for de novo lipogenesis and 
triglyceride synthesis. Conversion of hypoxanthine/xanthine to uric acid by xanthine oxidase is associated with the generation of oxidants as hydrogen peroxide. Uric acid can form alkylating species by interacting with peroxynitrite. Soluble and crystallized uric acid decreases AMP-Kinase leading to decreased ability for fatty acid oxidation and triglyceride accumulation (Kuwabara et al., 2018). Hyperuricemia increases reactive oxygen species (ROS). ROS increase insulin receptor substrate phosphorylation at a serine residue and decreases its phosphorylation at a tyrosine residue so two reactions block the phosphorylation of protein kinase $\mathrm{B}$ at a serine residue. Hyperuricemia decreases endothelial NO production which leads to decrease blood flow and glucose uptake by cells (Wang et al., 2018).

\section{Conclusions}

From the observations of the study performed, it could be predicted that fructose sweetened beverages and fats in our diet is shared occurrence of MetS. Menopause is aggravating MetS. Febuxostat can prevent pathological condition of MetS by different mechanisms of action and depends on duration of treatments.

\section{References}

Bayne K (1996). Revised Guide for the Care and Use of Laboratory Animals available. American Physiological Society. Physiologist, 39(4):199-211.

Cai S,Ou Z, Liu D, Liu L, Liu Y, Wu X, et al. (2018). Risk factors associated with liver steatosis and fibrosis in chronic hepatitis B patient with component of metabolic syndrome. United European Gastroenterology Journal, 6(4):558-566. doi: $10.1177 / 2050640617751252$

Caliceti C, Calabria D, Roda A, Cicero A. (2017). Fructose Intake, Serum Uric Acid, and Cardiometabolic Disorders: A Critical Review. Nutrients, 9(4): 395. doi: 10.3390/nu9040395

Das Undurti N. (2016). Renin-angiotensinaldosterone system in insulin resistance and metabolic syndrome. Journal of translational internal medicine, 4(2): 6672. doi: 10.1515/jtim-2016-0022

Drabsch T, Holzapfel C, Stecher L, Petzold J, Skurk T,Hauner, H. (2018). Associations Between C-Reactive Protein, Insulin Sensitivity, and Resting Metabolic Rate in Adults: A Mediator Analysis. Frontiers in Endocrinology, 9(556). doi: 10.3389/fendo.2018.00556

Er L K, Wu S, Chou H H, Hsu L A, Teng M S, Sun Y C, et al. (2016). Triglyceride Glucose-Body Mass Index Is a Simple and Clinically Useful Surrogate Marker for Insulin Resistance in Nondiabetic Individuals. PLoS One, 11(3): e0149731. doi: 10.1371.

Feillet-Coudray C, Fouret G, Vigor C, Bonafos B, Jover B, BlachnioZabielska A, et al. (2019). Long-Term Measures of Dyslipidemia, Inflammation, and Oxidative Stress in Rats Fed a High-Fat/High-Fructose Diet. Lipids, 54(1): 81-97. doi: 10.1002/lipd.12128

Heikal M M, Shaaban A A, Elkashef W F,Ibrahim T M. (2019). Effect of febuxostat on biochemical parameters of hyperlipidemia induced by a high-fat diet in rabbits. Canadian journal of physiology and pharmacology, 97(7): 611-622. doi: 10.1139/cjpp-2018-0731.

Khajuria D K., Razdan R and Mahapatra D R. (2012). Description ofa new method of ovariectomy in female rats.

RevistaBrasileira deReumatologia, 52: 466-470.

Kozakowski J, Gietka-Czernel M, Leszczyńska D,Majos A. (2017). Obesity in menopause - our negligence or an unfortunate inevitability? Przeglad menopauzalny. Menopause review, 16(2):61-65. doi:10.5114/pm.2017.68594. 
Lozano I, Van der Werf R, Bietiger W, Seyfritz E, Peronet C, Pinget $M$, et al. (2016). High-fructose and high-fat dietinduced disorders in rats: impact on diabetes risk, hepatic and vascular complications. Nutrition \& Metabolism, 13(1): 15. doi: 10.1186/s12986-0160074-1.

Luna G, Dolzhenko A V,Mancera $R$ L. (2019). Inhibitors of xanthine oxidase: scaffold diversity and structure-based drug design. ChemMedChem, 14(7): 714-743.

Meng J, Li Y, Yuan X. (2017). Effects of febuxostat on insulin resistance and expression of high-sensitivity C-reactive protein in patients with primary gout. Rheumatology International, 37(2): 299303. doi: 10.1007/s00296-016-3612-2.

Omizo H, Tamura Y, Morimoto C, Ueno M, Hayama Y,Kuribayashi-Okuma E,et al. (2020). Cardio-renal protective effect of the xanthine oxidase inhibitor febuxostat in the 5/6 nephrectomy model with hyperuricemia. Scientific Reports, 10(1): 9326. doi: 10.1038/s41598-02065706-6

Pimenta F, Tose H, Waichert É, Jr da Cunha M, Campos F V, VasquezE C, et al. (2019). Lipectomy associated to obesity produces greater fat accumulation in the visceral white adipose tissue of female compared to male rats. Lipids in Health and Disease, 18(1): 18-44 doi: 10.1186/s12944-0190988-5

\section{Rochlani Y, Pothineni N V, Kovelamudi S,Mehta J L (2017). Metabolic syndrome: pathophysiology, management, and modulation by natural compounds. Therapeutic advances in cardiovascular disease, 11(8): 215-225. doi: $10.1177 / 1753944717711379$}

Saklayen M G. (2018). The Global Epidemic of the Metabolic Syndrome. Current Hypertension Reports, 20(2): 12. doi: 10.1007/s1 1906-018-0812-z

Shirakura T, Nomura J, Matsui C, Kobayashi T, Tamura M, Masuzaki H.(2016). Febuxostat, a novel xanthine oxidoreductase inhibitor, improves hypertension and endothelial dysfunction in spontaneously hypertensive rats. Naunyn-Schmiedeberg's archives of pharmacology, 389(8): 831-838.

Stefanska A, Bergmann K,Sypniewska G. (2015). Metabolic Syndrome and Menopause: Pathophysiology, Clinical and Diagnostic Significance. Advances in clinical chemistry, 72: 1-75.doi: 10.1016/bs.acc.2015.07.001

Suckow Mark A, Weisbroth Steven H, Franklin Craig L. (2005). The laboratory rat: Elsevier.

Taskinen M R, Packard C J, Borén J. (2019). Dietary Fructose and the Metabolic Syndrome. Nutrients, 11(9): 1987. doi: 10.3390/nu11091987

Techatraisak K,Kongkaew T. (2017). The association of hyperuricemia and metabolic syndrome in Thai postmenopausal women. Climacteric, 20(6): 552-557.

Wang H, Zhang H, Sun L,Guo W. (2018). Roles of hyperuricemia in metabolic syndrome and cardiac-kidney-vascular system diseases. American journal of translational research, 10(9): 2749-2763.

Zhuhua Z, Zhiquan W, Zhen Y, Yixin N, Weiwei Z, Xiaoyong L, et al. (2015). A novel mice model of metabolic syndrome: the high-fat-high-fructose diet-fed ICR mice. Experimental animals, 64(4): 435-442. doi: 10.1538/expanim.14-0086 